\title{
A Simple Differentiation Protocol for Generation of Induced Pluripotent Stem Cell-Derived Basal Forebrain-Like Cholinergic Neurons for Alzheimer's Disease and Frontotemporal Dementia Disease Modeling
}

\author{
Sonia Sanz Muñoz ${ }^{1,2}\left(\right.$, Martin Engel ${ }^{1,2}\left(\mathbb{D}\right.$, Rachelle Balez ${ }^{1,2}\left({ }^{1}\right.$, Dzung Do-Ha ${ }^{1,2}$, \\ Mauricio Castro Cabral-da-Silva ${ }^{1,2} \mathbb{D}^{\mathbb{D}}$, Damian Hernández ${ }^{3,4,5}$, Tracey Berg ${ }^{1,2}$, Jennifer A. Fifita ${ }^{6}$, \\ Natalie Grima ${ }^{6}{ }^{(0)}$, Shu Yang ${ }^{6}$, Ian P. Blair ${ }^{6}$, Garth Nicholson ${ }^{7}$, Anthony L. Cook ${ }^{8}$, \\ Alex W. Hewitt $4,5,9$ (D), Alice Pébay ${ }^{3,4,5}$ (D) and Lezanne Ooi 1,2,*(D) \\ 1 Illawarra Health and Medical Research Institute, Wollongong, NSW 2522, Australia; \\ ssm886@uowmail.edu.au (S.S.M.); mengel@uow.edu.au (M.E.); rb478@uowmail.edu.au (R.B.); \\ pddh859@uowmail.edu.au (D.D.-H.); mcastro@uow.edu.au (M.C.C.-d.-S.); tberg@uow.edu.au (T.B.) \\ 2 School of Chemistry and Molecular Bioscience, University of Wollongong, Wollongong, NSW 2522, Australia \\ 3 Department of Anatomy \& Neuroscience, University of Melbourne, Parkville, VIC 3010, Australia; \\ Damian.Hernandez@unimelb.edu.au (D.H.); apebay@unimelb.edu.au (A.P.) \\ 4 Department of Surgery, University of Melbourne, Parkville, VIC 3010, Australia; Alex.Hewitt@utas.edu.au \\ 5 Centre for Eye Research Australia, Royal Victoria Eye and Ear Hospital, Melbourne, VIC 3002, Australia \\ 6 Centre for Motor Neuron Disease Research, Department of Biomedical Sciences, Faculty of Medicine and \\ Health Sciences, Macquarie University, Sydney, NSW 2190, Australia; jennifer.fifita@mq.edu.au (J.A.F.); \\ natalie.grima@mq.edu.au (N.G.); shu.yang@mq.edu.au (S.Y.); ian.blair@mq.edu.au (I.P.B.) \\ 7 Concord Repatriation General Hospital, University of Sydney ANZAC Research Institute, Sydney, \\ NSW 2006, Australia; garth.nicholson@sydney.edu.au \\ 8 Wicking Dementia Research and Education Centre, University of Tasmania, Hobart, TAS 7001, Australia; \\ anthony.cook@utas.edu.au \\ 9 School of Medicine, Menzies Institute for Medical Research, University of Tasmania, Hobart, \\ TAS 7000, Australia \\ * Correspondence: lezanne@uow.edu.au; Tel.: +61-24-221-5865
}

Received: 3 August 2020; Accepted: 29 August 2020; Published: 2 September 2020

\begin{abstract}
The study of neurodegenerative diseases using pluripotent stem cells requires new methods to assess neurodevelopment and neurodegeneration of specific neuronal subtypes. The cholinergic system, characterized by its use of the neurotransmitter acetylcholine, is one of the first to degenerate in Alzheimer's disease and is also affected in frontotemporal dementia. We developed a differentiation protocol to generate basal forebrain-like cholinergic neurons (BFCNs) from induced pluripotent stem cells (iPSCs) aided by the use of small molecule inhibitors and growth factors. Ten iPSC lines were successfully differentiated into BFCNs using this protocol. The neuronal cultures were characterised through RNA and protein expression, and functional analysis of neurons was confirmed by whole-cell patch clamp. We have developed a reliable protocol using only small molecule inhibitors and growth factors, while avoiding transfection or cell sorting methods, to achieve a BFCN culture that expresses the characteristic markers of cholinergic neurons.
\end{abstract}

Keywords: induced pluripotent stem cells; disease modelling; neuronal differentiation; cholinergic neurons; Alzheimer's disease; frontotemporal dementia 


\section{Introduction}

The use of induced pluripotent stem cells (iPSCs) has opened the possibility to generate relevant cell types that contain the genetic background of each donor, allowing the study of neurodegenerative diseases in a reductionist system. The cholinergic system is affected in dementias, including Alzheimer's disease (AD) and frontotemporal dementia (FTD) [1,2]. AD is a progressive and irreversible neurodegenerative disease characterized by cognitive impairment and memory loss. Currently, the drugs that are prescribed to AD patients target symptoms and show limited efficacy [3]. $\mathrm{AD}$ patients undergo neurodegeneration throughout the brain, however basal forebrain-like cholinergic neurons (BFCNs), part of the cholinergic system and characterized by the use of the neurotransmitter acetylcholine (ACh), are the first type of neurons to degenerate [1,2]. Basal forebrain atrophy also occurs in FTD, with degeneration of the cholinergic system thought to be causative in learning, and memory in FTD patients [4,5]. The potential selective vulnerability of the BFCNs, however has to date been difficult to study in human neurons.

$\mathrm{AD}$ can be divided into two forms: familial $\mathrm{AD}(\mathrm{FAD})$, also known as early-onset $\mathrm{AD}$, and sporadic $\mathrm{AD}(\mathrm{SAD})$, also known as late-onset $\mathrm{AD}$. FAD makes up less than $5 \%$ of all cases, and it is characterised by mutations in the amyloid precursor protein (APP) or presenilin (PSEN) genes, both of which are involved in amyloid $\beta(\mathrm{A} \beta)$ processing [6]. In contrast, SAD is the most common form, where the possession of the $\varepsilon 4$ allele of apolipoprotein $\mathrm{E}(A P O E)$ gene is the major genetic risk factor [7-9], but aging, environmental and lifestyle factors also influence the risk.

To ensure successful differentiation of BFCNs in vitro, a combination of growth factors and small molecule inhibitors mimicking in vivo BFCN development are important, and very few protocols have been developed, as reviewed in [10]. The published protocols to generate BFCNs used a combination of adherent and floating cultures, also involving transfection and cell sorting based purification, which dramatically affects yields [11-13]. Differences in BFCN purity and efficiency (based on choline acetyl transferase (ChAT) positive cells) have been identified in the published protocols, which is a major challenge to maintaining experimental consistency when multiple cell lines are compared.

The aim of the present study was to generate and characterise a robust protocol to differentiate a pure culture of BFCNs from iPSCs and validate it by gene expression and functional characterisation. The model described provides a relevant cell type to study AD pathways and for drug discovery.

\section{Materials and Methods}

\section{1. iPSC Lines and iPSC Maintenance}

The details of the iPSC lines used in this study are described in Table 1.

Human feeder-free iPSCs were cultured on Matrigel (Corning, \#354277) coated $60 \mathrm{~mm}$ diameter tissue culture dishes fed with TeSR-E8 Basal Medium (Stemcell Technologies, \#5990) and kept in a humidified incubator at $37{ }^{\circ} \mathrm{C}, 5 \% \mathrm{CO}_{2}$ and $3 \% \mathrm{O}_{2}$, mimicking the physiological oxygen levels in the brain. After manual removal of spontaneously differentiating cells under the microscope, the medium was changed every day. Colonies were passaged every 5-6 days using $1 \mathrm{U} / \mathrm{mL}$ dispase (Stemcell Technologies, \#7913) for $5 \mathrm{~min}$ at $37^{\circ} \mathrm{C}$. Once the border of the colonies started to detach from the surface, cells were washes off twice with Dubelcco's modified Eagle medium/Nutrient Mixture F-12 (DMEM/F12). DMEM/F12 was added a third time, colonies were mechanically detached with a cell scraper, transferred into a conical centrifuge tube and centrifuged for $5 \mathrm{~min}$ at $300 \times g$. The supernatant was removed, and colonies were gently resuspended in $1 \mathrm{~mL}$ TeSR-E8 before being plated down at a 1:5 ratio. The iPSC cultures were tested routinely for mycoplasma and karyotyping of all the iPSC lines was performed within 20 passages before the start of the differentiation. 
Table 1. List of iPSC lines used. The iPSC lines with the name used, sex and APOE genotype. HC, Healthy Control, SAD, Sporadic Alzheimer's disease, FAD, Familial Alzheimer's disease, ALS, Amyotrophic Lateral Sclerosis, FTD, Frontotemporal Dementia.

\begin{tabular}{ccccc}
\hline Name & iPSC Line & Sex & APOE & Reference \\
\hline HC1 & $1-4$ & M & $\varepsilon 3 / 3$ & {$[14]$} \\
\hline HC2 & $6846.2($ UOWi002-A) & F & $\varepsilon 2 / 3$ & {$[15]$} \\
\hline HC3 & RB9-8 (UOWi001-A) & F & $\varepsilon 2 / 4$ & {$[16]$} \\
\hline HC4 & MBE60-1 & M & $\varepsilon 3 / 3$ & Figure S1 \\
\hline HC5 & MBE68-1 & F & $\varepsilon 3 / 3$ & {$[17]$} \\
\hline SAD1 & RB7-11 (UOWi006-A) & F & $\varepsilon 4 / 4$ & Figure S2 \\
\hline SAD2 & $8-5$ & M & $\varepsilon 4 / 4$ & {$[18]$} \\
\hline SAD3 & $10-13$ & M & $\varepsilon 3 / 4$ & {$[14]$} \\
\hline FAD1 & $6848.2($ UOWi003-A) & F & $\varepsilon 3 / 4$ & {$[15]$} \\
\hline ALS/FTD1 & C-10 (UOWi008-A) & F & $\varepsilon 3 / 3$ & Figure S3
\end{tabular}

\subsection{Generation of Forebrain Cholinergic Neurons}

Following iPSC culture, TeSR-E8 medium was changed to Neural induction (Ni) medium $(0.4 \%$ (v/v) B27 supplement (Life Technologies (Carlsbad, CA, U.S.A.), \#17504044), 1\% (v/v) N2 supplement (Life Technologies, \#17502048), 1\% (v/v) Non-essential amino acids (Life Technologies, \#11140050) and 1\% (v/v) GlutaMAX (Life Technologies, \#35050061) in DMEM/F12) supplemented with $0.1 \mu \mathrm{M}$ LDN193189 (Focus Bioscience, \#12071A), a Bone morphogenic protein (BMP) inhibitor 2 days before passaging. Medium was changed if necessary, depending on the density of the cultures, avoiding acidic conditions.

On the passaging day, cells were incubated with $1 \mathrm{U} / \mathrm{mL}$ dispase until colonies fully detached from the plate. Colonies were collected and rinsed twice with DMEM/F12 to remove the enzyme, allowing them to settle by gravity for $2 \mathrm{~min}$ at $22{ }^{\circ} \mathrm{C}$. The supernatant was removed, and colonies were resuspended in Ni media supplemented with $0.1 \mu \mathrm{M}$ LDN193189 and $10 \mu \mathrm{M}$ of the Transforming Growth Factor (TGF)- $\beta$ inhibitor SB431542 (Focus Bioscience, \#10431), pipetting gently up and down to break down the colonies. Colonies were transferred into a low adherent non-tissue culture plate to form floating embryoid bodies (EBs). Full medium change with Ni media containing $0.1 \mu$ M LDN193189 and $10 \mu \mathrm{M} \mathrm{SB} 431542$ was performed every second day.

EBs were collected on day 5 and plated down into Matrigel-coated tissue culture plates with $\mathrm{Ni}$ media supplemented with $10 \mathrm{ng} / \mathrm{mL}$ Fibroblast Growth Factor (FGF)-2 (Global Stem, \#GDR-2001) to form neural rosettes. A partial volume media change with Ni medium supplemented with $10 \mathrm{ng} / \mathrm{mL}$ FGF-2 was performed after $48 \mathrm{~h}$ and with $10 \mathrm{ng} / \mathrm{mL}$ FGF-2 and $50 \mathrm{ng} / \mathrm{mL}$ SHH (Stemcell Technologies, \#78075) after $96 \mathrm{~h}$. Neural rosette formations were visible by day 7.

Once neural rosettes were visible on day 7, medium was removed, and cells were incubated with $1 \mathrm{U} / \mathrm{mL}$ dispase until the colony edges loosened. Dispase was removed and cells were carefully rinsed 3 times with DMEM/F12 to ensure the removal of dispase and cell debris. Neural expansion (Ne) medium ( $2 \%(v / v)$ B27 supplement, $1 \%(v / v)$ N2 supplement, $1 \%(v / v)$ Non-essential amino acids and $1 \%(v / v)$ GlutaMAX in DMEM/F12) supplemented with $100 \mathrm{ng} / \mathrm{mL}$ Sonic Hedgehog (SHH) was added and identified neural rosettes were detached by aspiration using a P1000 pipette. Floating neural rosettes were transferred into a low adherent non-tissue culture plate to form floating neurospheres. Half volume media changes were performed every second day using Ne medium supplemented with $100 \mathrm{ng} / \mathrm{mL}$ SHH for the first 6 days; $100 \mathrm{ng} / \mathrm{mL}$ SHH and $100 \mathrm{ng} / \mathrm{mL}$ FGF-8 (Stemcell Technologies, \#78128) from day 6 until day 12; and 100 ng/mL SHH, 100 ng/mL FGF-8 and 10 ng/mL BMP9 (Peprotech, \#120-07) on day 12. 
On day 15 of neurosphere stage, neurospheres were washed twice with DMEM/F12 prior dissociation with StemPro Accutase (Life Technologies, \#A1110501) for $20 \mathrm{~min}$ at $37^{\circ} \mathrm{C}$. Enzymatic dissociation was stopped by dilution with DMEM/F12 and cells were recovered by centrifugation at $300 \times \mathrm{g}$ for $5 \mathrm{~min}$. Cells were counted and 125,000 cells/well were plated on Matrigel and $0.1 \mathrm{mg} / \mathrm{mL}$ collagen I (Thermo Fisher Scientific, \#A1048301) -coated 24-well plate format.

Neuronal maturation (Nm) medium (1\% (v/v) B27 supplement and 1\% $(v / v)$ GlutaMAX in Brainphys (Stemcell Technologies, \#5790)) supplemented with $100 \mathrm{ng} / \mathrm{mL}$ of SHH, $100 \mathrm{ng} / \mathrm{mL}$ of FGF-8, $10 \mathrm{ng} / \mathrm{mL}$ of BMP9 and $100 \mathrm{ng} / \mathrm{mL}$ of Nerve Growth Factor (NGF) (Peprotech, \#450-01) was used on days 1 and 3 of cholinergic maturation. On day 5, a half volume media change using Nm media supplemented with $5 \mathrm{ng} / \mathrm{mL}$ of BDNF (Miltenyi Biotech, \#130-093-811) and $100 \mathrm{ng} / \mathrm{mL}$ of NGF in addition to $1 \mu \mathrm{M}$ 5-Fluoro-2'-deoxyuridine (5FdU, Sigma-Aldrich, \#F0503) to stop the proliferation of the dividing cells was used. A full media change was performed on day 7 using Nm medium supplemented with $5 \mathrm{ng} / \mathrm{mL}$ Brain Derived Neurotrophic Factor (BDNF) and $100 \mathrm{ng} / \mathrm{mL}$ NGF. Partial volume media changes using Nm medium with $5 \mathrm{ng} / \mathrm{mL}$ BDNF and $10 \mathrm{ng} / \mathrm{mL}$ NGF were performed every second day for a total of 4 weeks to allow neuronal maturation.

\subsection{Cell Imaging and Immunostaining}

At week 4 of neuronal maturation, $\mathrm{Nm}$ medium was removed, neurons were rinsed with phosphate-buffered saline (PBS) and fixed by incubation with $4 \%(w / v)$ paraformaldehyde (PFA) at room temperature. After $10 \mathrm{~min}$, PFA was removed and cells were rinsed three times with PBS, permeabilised with $0.5 \%(v / v)$ Triton X-100 in PBS for $15 \mathrm{~min}$ and rinsed three times with PBS. Blocking was performed with $5 \%(v / v)$ goat serum in PBS and $0.3 \mathrm{M}$ glycine for $1 \mathrm{~h}$ at $22{ }^{\circ} \mathrm{C}$. Primary antibodies were used at the specific dilution in $5 \%(v / v)$ goat serum in PBS and incubated at $4{ }^{\circ} \mathrm{C}$ for $16 \mathrm{~h}$. On the following day, cells were rinsed three to five times with PBS and incubated with the appropriate secondary antibody made up in PBS for $1 \mathrm{~h}$ at $22^{\circ} \mathrm{C}$. Cells were washed three to five times with PBS before incubation with Hoechst 33,342 (Thermo Fisher Scientific, \#62249, $1 \mu \mathrm{g} / \mathrm{mL}$ ) or Reddot 2 (Biotium, \#40061-T, 1:200) for 10 or 20 min respectively for nuclear staining. Coverslips were mounted on glass slides using ProLong Gold antifade reagent (Life Technologies, \#P10144). Confocal microscopy was performed using Leica TCS SP8 (Leica Microsystems, Germany) microscopy images were analysed using Leica Application Suite - Advanced Fluorescence (LAS-AF) software. The antibodies used for immunocytochemistry are listed in Table 2.

Table 2. List of antibodies used for immunocytochemistry.

\begin{tabular}{cccc}
\hline Antibody & Raised in & Dilution & Company Cat \# \\
\hline Anti- $\beta$-III-tubulin & Mouse mAb & $1: 1000$ & Abcam, \#ab78078 \\
\hline Anti-ChAT & Rabbit pAb & $1: 100$ & Abcam, \#ab181023 \\
\hline Anti-p75 & Rabbit mAb & $1: 50$ & Abcam, \#ab52987 \\
\hline Anti-GFAP & Rabbit mAb & $1: 500$ & Millipore, \#04-1062 \\
\hline Anti-MAP2 & Mouse mAb & $1: 500$ & Millipore, \#MAB3418 \\
\hline $\begin{array}{c}\text { Anti-Rabbit IgG }(\mathrm{H}+\mathrm{L}) \\
\text { Alexa Fluor 488 }\end{array}$ & Goat pAb & $1: 1000$ & Life Technologies, \#A11008 \\
\hline $\begin{array}{c}\text { Anti-Mouse IgG }(\mathrm{H}+\mathrm{L}) \\
\text { Alexa Fluor 633 }\end{array}$ & Goat pAb & $1: 1000$ & Life Technologies, \#A21050 \\
\hline
\end{tabular}

\subsection{Nanostring}

A custom CodeSet was designed to be used with the PlexSet Nanostring technology (Nanostring) to analyse the genes in Table S1. The mRNA from iPSC and BFCN samples was harvested in TriSURE (Bioline, \#38032) and extracted following the manufacturer's instructions. The mRNA concentration of 
the samples was measured using a Qubit 3.0 Fluorometer (Thermo Fisher Scientific). To determine the optimal amount of mRNA per sample needed to not saturate the Nanostring, a titration run was performed and analysed using the nSolver System (Nanostring). A total of $100 \mathrm{ng}$ mRNA per sample were run in the nCounter SPRINT (Nanostring). To normalize the samples, a reference sample compiled of all the samples was run alongside each Probe Set, and a total of 10 housekeepers (AARS, ASB7, CCDC127, CNOT10, EID2, MTO1, RABEP2, SUPT7L, TADA2B, ZNF324B) were selected based on the literature and Nanostring recommendations. Samples generated were analysed for each gene independently showing the number of molecules counted by the nCounter SPRINT System after normalization by housekeepers using the nSolver System.

\subsection{Whole Cell Patch Clamp}

Whole-cell patch-clamp was performed on the BFCNs after 4 weeks of maturation to assess the neuronal functionality. All experiments were performed at $22-24{ }^{\circ} \mathrm{C}$ in artificial cerebral spinal fluid (aCSF, $135 \mathrm{mM} \mathrm{NaCl}, 5 \mathrm{mM} \mathrm{KCl}, 2 \mathrm{mM} \mathrm{CaCl} 2,1 \mathrm{mM} \mathrm{MgCl} 2,10 \mathrm{mM}$ HEPES and $10 \mathrm{mM}$ D-glucose; adjusted to $\mathrm{pH} 7.4$ (with $\mathrm{NaOH}$ ) and $\sim 305 \mathrm{mOsm}$ ) and using intracellular solution (150 $\mathrm{mM} \mathrm{KCl}$, $2 \mathrm{mM} \mathrm{MgCl}$, $10 \mathrm{mM}$ HEPES, $4 \mathrm{mM}$ Mg-ATP, $0.3 \mathrm{mM}$ Na-GTP, $10 \mathrm{mM}$ Na2PCr and $1 \mathrm{mM}$ EGTA; adjusted to $\mathrm{pH} 7.4$ (with $\mathrm{KOH}$ ) and $\sim 295 \mathrm{mOsm}$ ). Cells were visualised with an inverted microscope (Leica DM RB) and chosen based on neuronal morphology, which included large cell bodies and long extensions. Patching electrodes were pulled by a Sutter P97 from a $1.5 \mathrm{~mm}$ OD $\times 0.86 \mathrm{~mm}$ ID borosilicate glass (with filament) (Harvard Apparatus) to a resistance of 5-7 M $\Omega$. Electrophysiological signals were recorded at $10 \mathrm{~Hz}$ using Digidata 1550 and amplified using a MultiClamp 700B amplifier (Axon Instruments, Molecular Devices Electrophysiology). The acquired data were analysed using the pCLAMP10 software. Whole-cell patch clamp configuration was achieved after establishing a $\mathrm{G} \Omega$ seal $(>1 \mathrm{G} \Omega$ ). Cells with a series resistance (Rs) below $25 \mathrm{M} \Omega$ were used for further analysis. Membrane Capacitance (CM), Rs and resting membrane potential (RMP) were determined using the Membrane Test and pClamp10 analysis software. To determine firing properties of differentiated neurons, direct current was injected to hold the cell at $-60 \mathrm{mV}$. In current clamp mode, step currents of $25 \mathrm{pA}$ were injected form $-50 \mathrm{pA}$ until the cell fired or $500 \mathrm{pA}$. Synaptic events were recorded in voltage clamp mode by injecting direct current, holding the cell at $-60 \mathrm{mV}$ and performing a chart recording over $5 \mathrm{~min}$.

\subsection{Data and Statistical Analysis}

Ten iPSC lines described in Table 1 were used for the optimization and characterization of the BFCN protocol. These lines include healthy controls, SAD, FAD and ALS/FTD lines in order to represent more variability in the population rather than several differentiations within each cell line.

The statistical analysis and graphs were performed using Prism 8 (GraphPad Prism 8). Each set of genes for iPSCs and BFCNs were checked for normality and therefore analysed using two-tailed $t$-test for those with parametric distribution and Kolmogorov-Smirnov for those with non-parametric distribution. The use of each test on the data is explained in the figure legends.

\section{Results}

The generation of BFCNs from iPSCs included adherent and suspension stages, with the addition of small molecule inhibitors and growth factors to mimic biochemical signals received during development. An overview of each stage of the protocol to generate BFCNs is represented in Figure 1. Incubation of iPSCs for $48 \mathrm{~h}$ prior to generation of embryoid bodies with Ni media supplemented with the BMP inhibitor LDN193189, was followed by the addition of LDN193189 and the TGF- $\beta$ inhibitor SB431542 in EB stage, in order to promote the ectoderm fate of the iPSCs. This could be observed by the formation of neural rosette-like structures inside the EBs (Figure 1C) and the generation of neural rosettes 5 days after plating the EBs onto Matrigel-coated plates (Figure 1D). Addition of FGF-2 in this stage stimulated neural progenitor expansion, and the formation of neural rosettes ensured the selection of 
the cells mimicking the neural tube formation by visual screening (Figure 1D). The neural rosettes were manually selected and kept in suspension to generate floating neurospheres. This structure allowed the expansion of the neural progenitors (Figure 1E) and, in the presence of $\mathrm{SHH}$ induces ventralization of the neural tube, the region from where BFCNs develop [19]. In the late stage of neurosphere expansion, the addition of FGF-8 mimicked the telencephalon development, and BMP9 induced the cholinergic phenotype of the progenitors [20]. As the last stage of the protocol, neurospheres were dissociated and plated as single cells, allowing the neural progenitors to mature as BFCNs (Figure 1F). Specific growth factors such as BDNF, which stimulates cholinergic differentiation, and NGF, which promotes neuronal maturation, arborization and survival, were required to promote the BFCN fate and increase ChAT activity [21-23]. The addition of 5-Fluoro-2' deoxyuridine (5FdU), which inhibits the proliferative cells by stopping DNA biosynthesis [24,25], helps the culture to focus on the furthest developed neurons and therefore can be used to generate highly pure neuronal cultures.

\section{A}

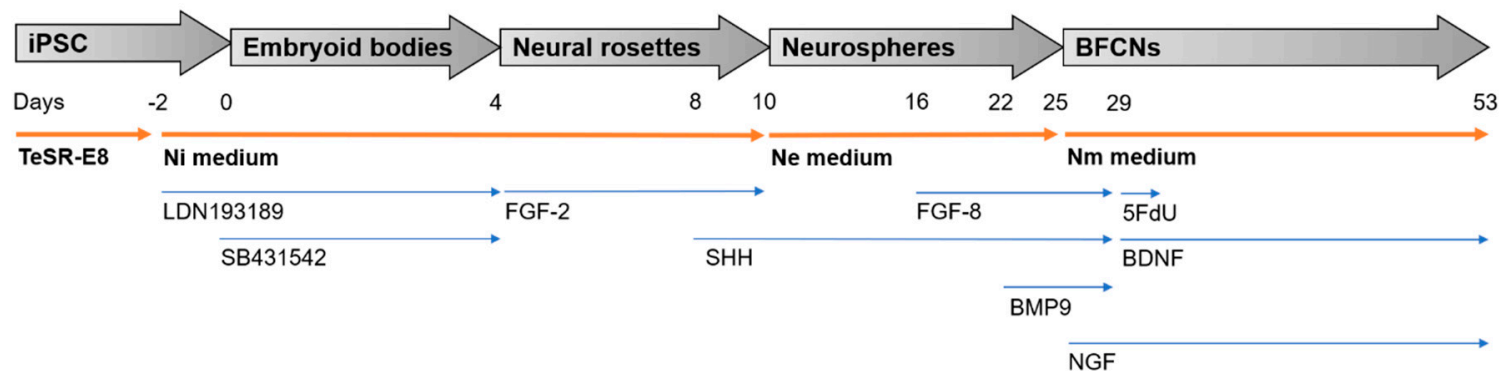
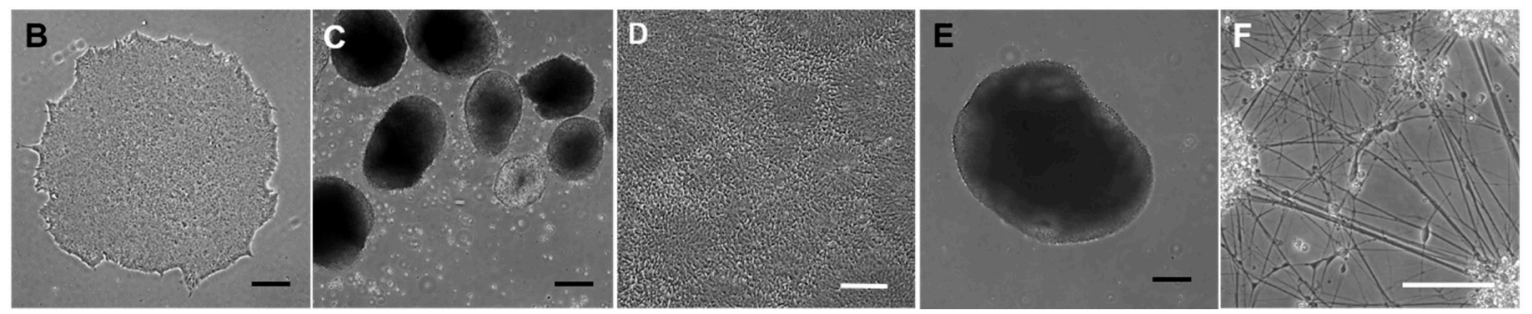

Figure 1. Differentiation of BFCNs from iPSCs. The iPSCs were differentiated into BFCNs by the combination of small molecule inhibitors and specific growth factors mimicking the developmental signals. (A) Schematic diagram showing the different stages of the differentiation in grey arrows over time. Orange arrows showing the cell culture media used in each stage and blue arrows showing the small molecule inhibitors or growth factors used at each specific time points. (B-F) Representative phase contrast pictures of the last day at each stage: (B) iPSC, (C) embryoid bodies, (D) neural rosettes, (E) neurospheres and (F) BFCNs. Black scale bars $=200 \mu \mathrm{m}$ and white scale bars $=100 \mu \mathrm{m}$.

Immunocytochemistry for general neuronal and specific cholinergic markers was performed at 4 weeks of maturation in BFCN cultures. The cholinergic marker ChAT and the neuronal marker $\beta$-III-tubulin were identified in the BFCN cultures. Visual counting showed that $87 \%$ of the $\beta$-III-tubulin positive cells were also ChAT positive (Figure $2 \mathrm{~A}-\mathrm{C}$ ). Immunocytochemistry against the NGF receptor p75NTR was also performed, with only a few cells identified as p75NTR+ve in the cultures and colocalised with $\beta$-III-tubulin (Figure 2D-F). The mRNA expression of the BFCN samples was analysed by Nanostring and compared to iPSC samples after adjusting to the expression of 10 housekeeping genes. The housekeepers were chosen to cover different ranges of expression (highly expressed, such as $A A R S$ or CCDC127, and lower expressed, such as RABEP2 or ZNF324B). The expression of each housekeeper gene is shown in Figure $S 4$ and there was no significant difference in the overall housekeeper expression between iPSCs and BFCNs (Figure S4). 

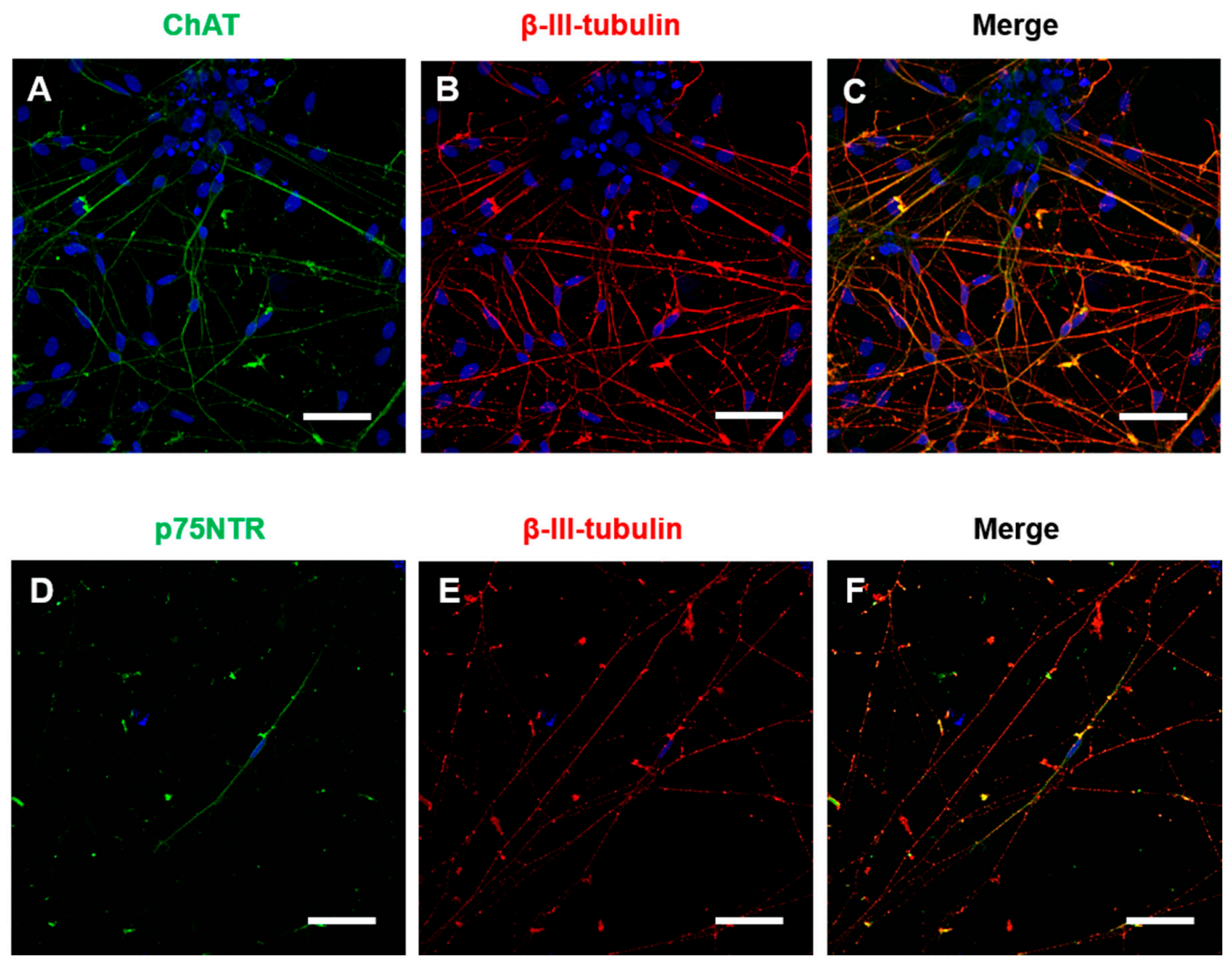

Figure 2. BFCNs were positive for neuronal markers. BFCN cultures were matured for 4 weeks and immunocytochemistry for general neuronal markers and specific cholinergic neuronal markers was performed. (A-F) Representative confocal pictures of the BFCN cultures at week 4 of BFCN maturation with the nuclear marker Hoechst 33,342 in blue. (A) Cholinergic marker ChAT in green. (B) Neuronal marker $\beta$-III-tubulin in red. (C) Merged picture of A and B. (D) Cholinergic marker p75NTR in green.

(E) Neuronal marker $\beta$-III-tubulin in red. (F) Merged picture of D and E. Scale bars $=50 \mu \mathrm{m}$.

To characterise the BFCN cultures, sets of genes for different stages of development were analysed, and their expression compared to iPSC cultures. As expected, the pluripotency markers NANOG and POU5F1 were highly expressed in iPSCs, whereas no expression was detected in BFCN cultures (Figure 3A,B). The RNA molecule counts for the pluripotency markers were in the range of 10,000-35,000 for all lines (Figure S5). The neuronal progenitor markers achaete-scute homolog 1 (ASCL1), distal-less homeobox 1 and 2 (DLX1 and DLX2), empty-spiracles homeobox 1 (EMX1), paired box 6 (PAX6) and SRY-box 1 (SOX1) were analysed (Figure 3C-H). EMX1 did not show a significant difference in expression between BFCN and iPSC cultures (Figure 3F). Assessing the RNA molecule count data showed that in all three SAD lines, but no other lines, an increase in EMX1 RNA was detected in the BFCNs, compared to iPSCs (Figure S6). However, all other neuronal progenitor genes were upregulated in BFCN cultures compared to iPSCs: ASCL1 was upregulated by $17.1 \pm 5.2$ fold $(p<0.001, \mathrm{D}=1)$, DLX1 was upregulated by $54 \pm 23$ fold ( $p=0.003, \mathrm{D}=0.8), D L X 2$ was upregulated by $34.6 \pm 10.7$ fold $(p<0.001, \mathrm{D}=1)$, PAX6 was upregulated by $16.6 \pm 4.3$ fold $(p=0.003, \mathrm{D}=0.8)$, and SOX1 was upregulated by $35.2 \pm 11$ fold $(p<0.001, \mathrm{D}=1)$. The specific $\mathrm{BFCN}$ progenitor markers analysed were FOXG1, ISL1, LHX8, and NKX2-1 (Figure 3I-L). While FOXG1, ISL1 and NKX2-1 were upregulated by $73.6 \pm 15.9$ fold $(p<0.001, \mathrm{D}=0.9), 53.3 \pm 17.2$ fold $(p<0.001, \mathrm{D}=0.9)$ and $7.2 \pm 3.7$ fold $(p=0.03$, $\mathrm{D}=0.8)$, respectively; $L H X 8$ did not show a significant difference between cultures. Of the BFCN progenitor markers, the most consistently upregulated gene across the 10 lines was FOXG1 (Figure S7). 
A

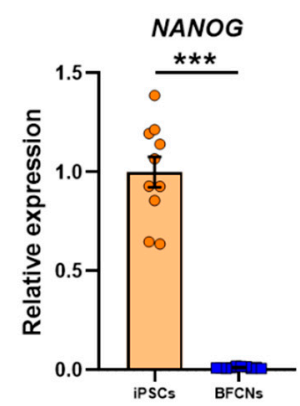

E

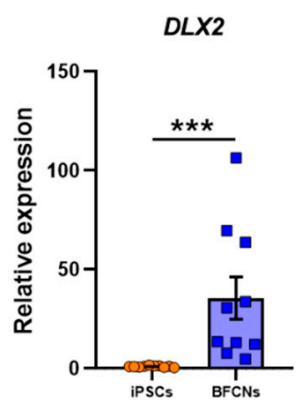

B

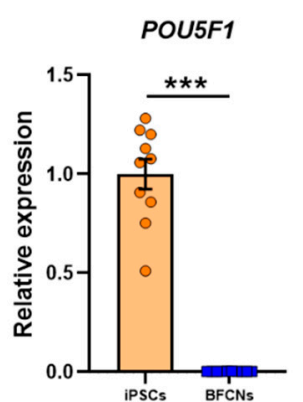

F

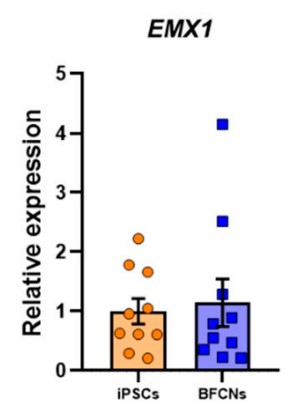

C

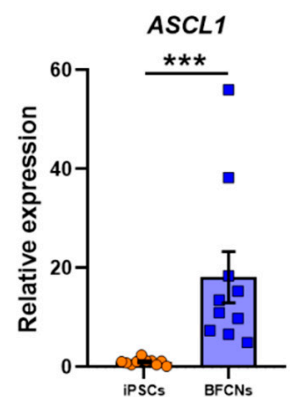

G

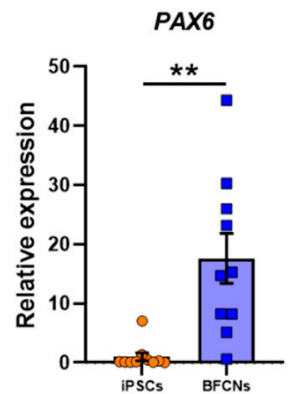

K

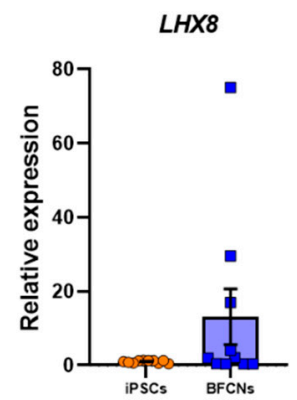

D

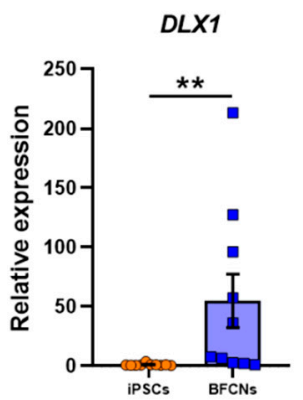

H sox1

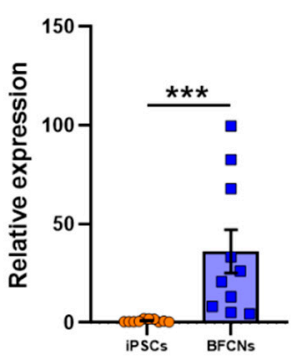

L

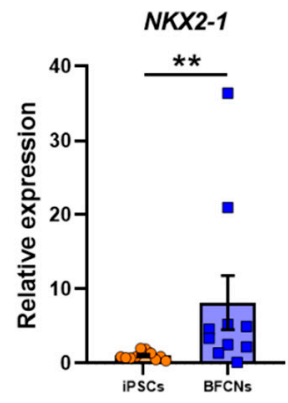

Figure 3. Expression of pluripotency, neuronal progenitors and specific cholinergic progenitor markers in iPSC and BFCN cultures. The iPSC and BFCN samples were analysed by nCounter (Nanostring) and, after normalisation of total amount of RNA molecules to the housekeeper genes, results are shown as fold change of BFCN cultures over iPSCs. Analysis of the pluripotency markers (A) NANOG and (B) POU5F1; the neuronal progenitor markers (C) ASCL1, (D) DLX1, (E) DLX2, (F) EMX1, (G) PAX6; and (H) SOX1; and the specific cholinergic progenitor makers (I) FOXG1, (J) ISL1, (K) LHX8 and (L) NKX2-1. Data are derived from 10 iPSC lines and 10 BFCN differentiated samples from 1 independent differentiation for each line. Individual data are shown. Histogram bars represent mean values and error bars represent S.E.M. Graphs show ${ }^{* *} p \leq 0.01{ }^{* * *} p \leq 0.001$, by Kolmogorov-Smirnov test except in $\mathrm{A}$ and $\mathrm{B}$ that two-tailed $t$-test was used for parametric distribution.

General neuronal markers such as solute carrier family 6 member 3 (SLC6A3, commonly known as dopamine transporter 1 (DAT1)), glutamate decarboxylase 2 (GAD2), glutamate ionotropic receptor AMPA type subunit 1 and 2 (GRIA1 and GRIA2), glutamate ionotropic receptor NMDA type subunit 1 (GRIN1), microtubule associated protein 2 (MAP2), disc large MAGUK scaffold protein 4 (DLG4, commonly known as postsynaptic density protein 95 (PSD95)), synapsin I (SYN1), tyrosine hydroxylase (TH) and $\beta$-III-tubulin (TUBB3) were also analysed in iPSC and BFCN cultures (Figure 4A-J). 
A

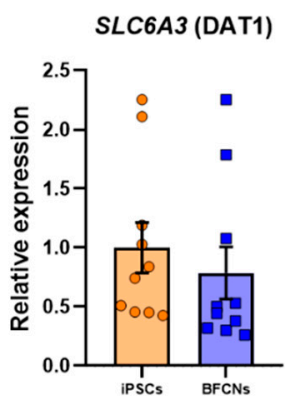

E

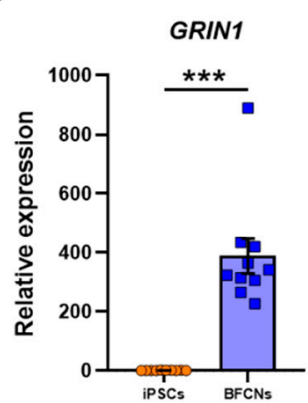

I

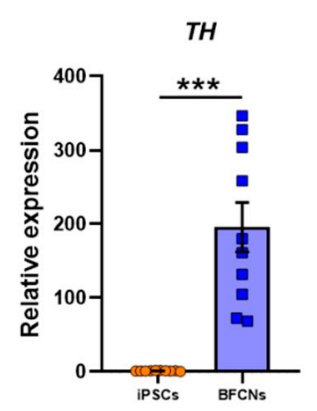

M

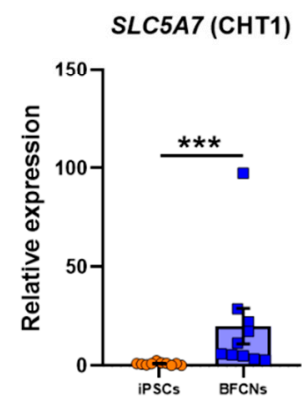

B

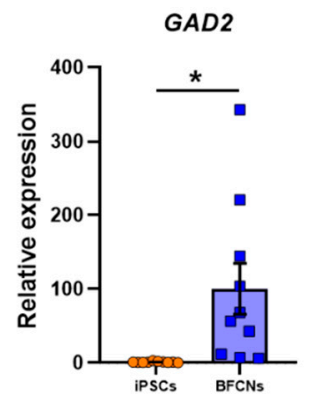

F

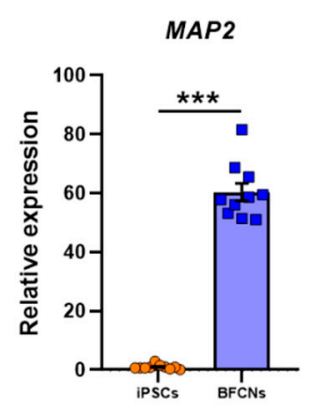

J

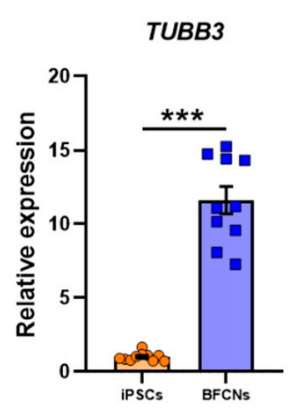

N

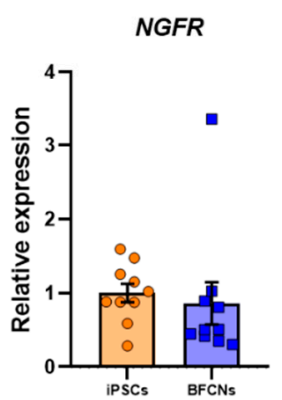

C

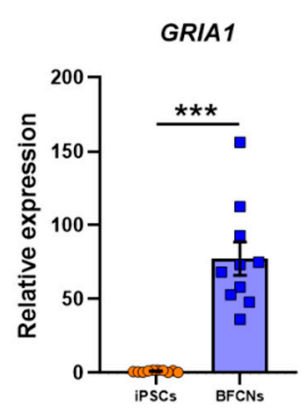

D

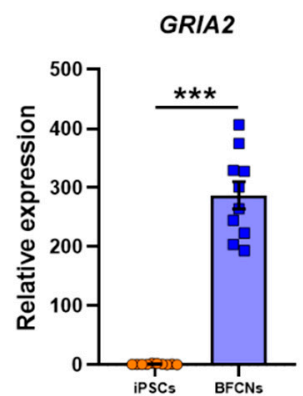

G

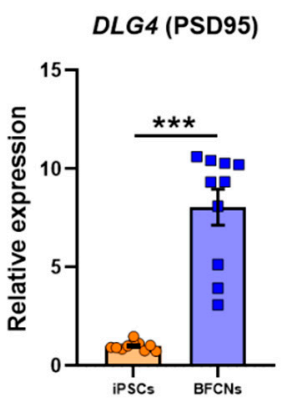

H

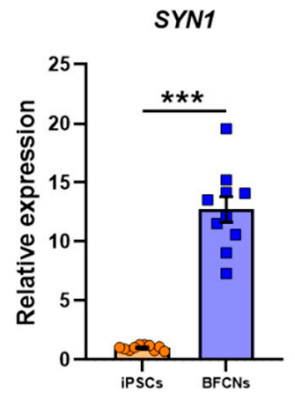

K

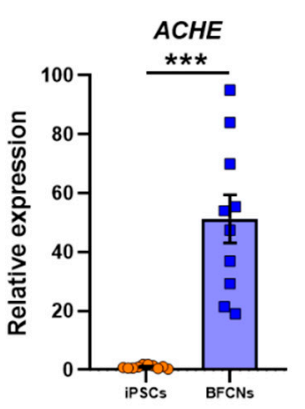

L

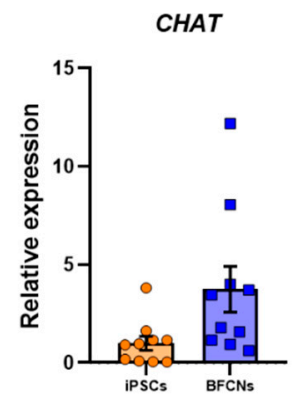

0

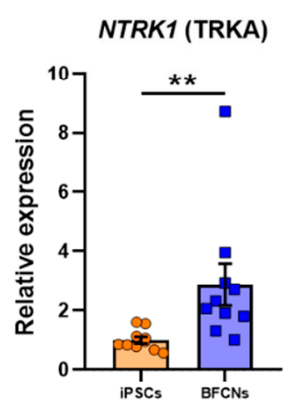

SLC18A3 (VACHT)

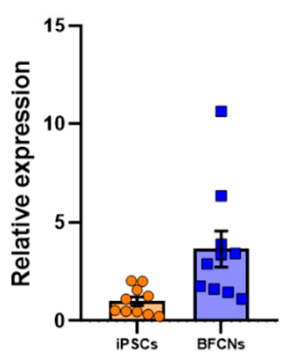

Figure 4. Expression of neuronal and specific BFCN markers in iPSC and BFCN cultures. The iPSC and BFCN samples were analysed by nCounter (Nanostring) and, after normalisation of total amount of RNA molecules to the housekeeper genes, results are shown as fold change of BFCN cultures over iPSCs. Analysis of the neuronal markers (A) SLC6A3 (DAT1), (B) GAD2, (C) GRIA1, (D) GRIA2, (E) GRIN1, (F) MAP2, (G) DLG4 (PSD95), (H) SYN1, (I) TH and (J) TUBB3; and the specific BFCN markers (K) ACHE, (L) CHAT, (M) SLC5A7 (CHT1), (N) NGFR, (O) NTRK1 (TRKA) and (P) SLC18A3 (VACHT). Data are derived from 10 iPSC lines and 10 BFCN differentiated samples from 1 independent differentiation for each line. Individual data are shown. Histogram bars represent mean values and error bars represent S.E.M. Graphs show ${ }^{*} p \leq 0.05,{ }^{* *} p \leq 0.01,{ }^{* * *} p \leq 0.001$, by two-tailed $t$-test except in A, D, E, G, L, M, N, O and P that Kolmogorov-Smirnov test was used for non-parametric distribution. 
The glutamate decarboxylase GAD2 and the glutamate receptors GRIA1, GRIA2 and GRIN1 were upregulated in BFCN when compared to iPSCs by $99.3 \pm 34.5$ fold $(p=0.01, \mathrm{t}=2.9), 76.3 \pm 11.2$ fold $(p<0.001, \mathrm{t}=6.8), 285.7 \pm 23$ fold $(p<0.001, \mathrm{D}=1)$ and $387.6 \pm 59.1$ fold $(p<0.001, \mathrm{D}=1)$, respectively. The RNA molecule counts across all 10 lines showed a consistent upregulation of the genes encoding the AMPA and NMDA receptor subunits, GRIA1, GRIA2, GRIN1 (5000-20,000 counts), as well as the neuronal cytoskeletal and synaptic protein genes, MAP2 (100,000-200,000 counts), TUBB3, DLG4 (PSD95) and SYN1 (10,000-40,000 counts; Figure S8). An upregulation of TH by $194.6 \pm 33.5$-fold $(p<0.001, \mathrm{t}=5.8)$ was also found in BFCN cultures, whereas the dopaminergic associated transporter DAT1 did not differ when compared to iPSCs. MAP2 and TUBB3, which had also been identified in the BFCN cultures by immunostaining (Figure 2B,E and Figure $5 \mathrm{~F}$ ), were upregulated by $59.3 \pm 3$ fold $(p<0.001, \mathrm{t}=19.91)$ and $10.6 \pm 0.9$ fold $(p<0.001, \mathrm{t}=11.45)$, respectively. Moreover, the pre-synaptic marker SYN1 and the post-synaptic marker PSD95 were upregulated by $11.7 \pm 1.1$-fold $(p<0.001, \mathrm{t}=10.73)$ and $7.05 \pm 0.9(p<0.001, \mathrm{D}=1)$, respectively. The cholinergic cell markers ACHE, CHAT, solute carrier family 5 member 7, (SLC5A7, commonly known as CHT1), nerve growth factor receptor (NGFR), neurotrophic receptor tyrosine kinase 1 (NTRK1, also known as TRKA) and solute carrier family 18 member 3 (SLC18A3, commonly known as VACHT) were analysed (Figure 4K-P). The cholinergic neuron marker $A C H E$ and $C H T 1$ were upregulated in BFCN cultures when compared to iPSCs by $50.2 \pm 8.2(p<0.001, \mathrm{t}=6.2)$ and $19 \pm 9.1(p<0.001, \mathrm{D}=1)$, respectively. However, the expression of CHAT and NGFR showed no significant difference between samples in the iPSC and BFCN cultures. The RNA molecule counts for $A C H E$ were more consistently upregulated across all 10 lines (5000-20,000 counts), compared to CHAT, SLC5A7 and SLC18A3 (Figure S9). Moreover, from the specific NGF receptors analysed, NTRK1 was upregulated by $1.9 \pm 0.7(p=0.003, \mathrm{D}=0.8)$, while NGFR did not show a significant difference when BFCNs were compared to iPSCs. However, iPSCs showed high RNA molecule expression in NGFR (Figure S9) and this gene was not further upregulated following differentiation into BFCNs. These data together confirm the cholinergic fate of the BFCN cultures.

To further characterize the purity of the BFCN cultures, the astrocyte markers aldolase (ALDOC), solute carrier family 1 member 3 (SLC1A3, commonly known as glial high affinity glutamate transporter 1 (EAAT1)), nuclear factor I A (NFIA) and S100B calcium binding protein B (S100B) were analysed (Figure 5A-D). ALDOC and NFIA did not show a significant difference when BFCN cultures were compared to iPSCs, whereas S100B was upregulated by $3.6 \pm 1.4(p<0.001, \mathrm{D}=0.9)$ and EAAT1 was downregulated by $0.6 \pm 0.3(p<0.001, \mathrm{D}=0.9)$. RNA molecule counts for the astrocyte marker genes were low in the majority of lines; in many but not all lines, the levels of the astrocyte marker genes were lower in BFCNs than in iPSCs (Figure S10). BFCN cultures were also positive for the dendritic marker microtubule-associated protein 2 (MAP2), but no astrocytes were detected after staining against the activated astrocyte marker Glial fibrillary acid protein (GFAP) (Figure $5 \mathrm{E}-\mathrm{G}$ ), indicative of a highly pure neuronal culture. 
A

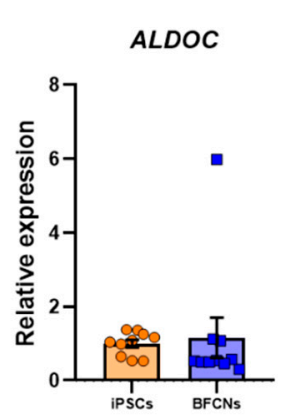

B

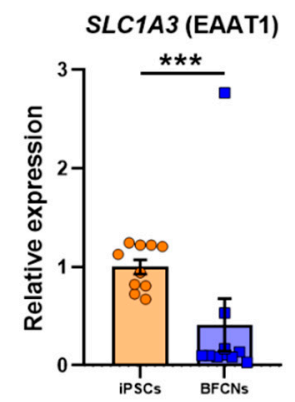

C

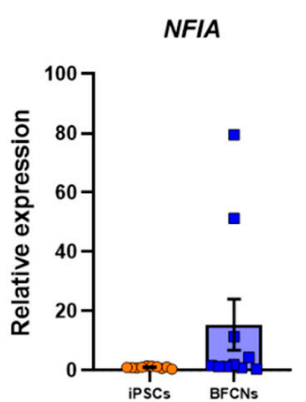

D

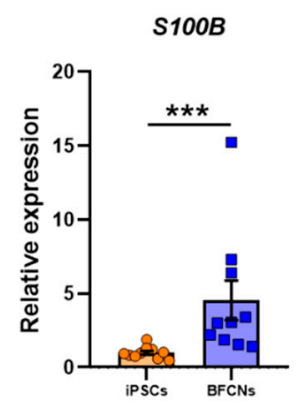

GFAP

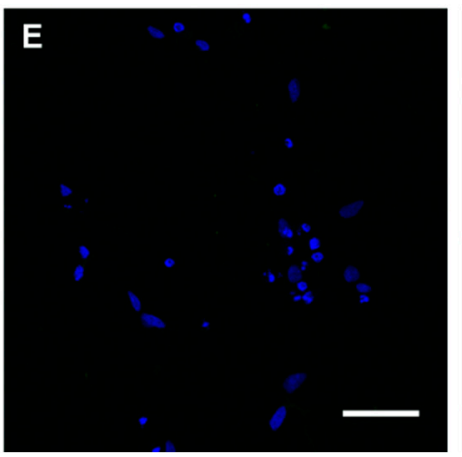

MAP2

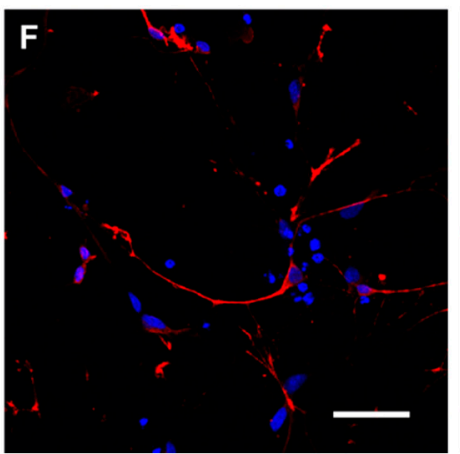

Merge

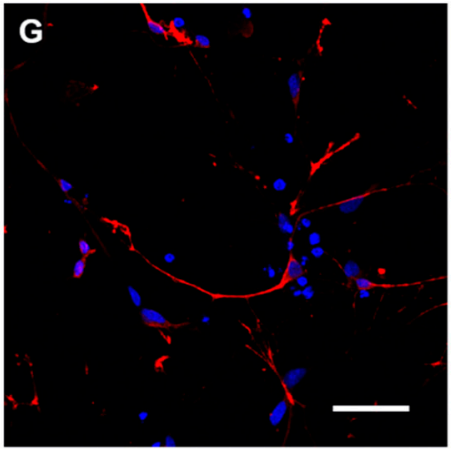

Figure 5. Expression of astrocytic markers. The iPSC and BFCN samples were analysed by nCounter (Nanostring) and, after normalisation of total amount of RNA molecules to the housekeeper genes, results are shown as fold change of BFCN cultures over iPSCs. Analysis of the astrocyte markers (A) ALDOC, (B) SLC1A3 (EAAT1), (C) NFIA and (D) S100B. Data are derived from 10 iPSC lines and $10 \mathrm{BFCN}$ differentiated samples from 1 independent differentiation for each line. Individual data are shown. Histogram bars represent mean values and error bars represent S.E.M. Graphs show ${ }^{* * *} p \leq 0.001$, by Kolmogorov-Smirnov test. BFCN cultures were matured for 4 weeks and immunocytochemistry for general neuronal markers and specific cholinergic neuronal markers was performed. (E-G) Representative confocal pictures of the BFCN cultures at week 4 of BFCN maturation with the nuclear marker Hoechst 33,342 in blue. (E) Astrocyte marker GFAP in green. (F) Dendritic marker MAP2 in red. (G) Merged picture of G and H. Scale bars $=50 \mu \mathrm{m}$.

Whilst the expression of protein markers was useful to validate cholinergic neuron gene expression, neurons are ultimately defined by their electrical properties. Consequently, we analysed the electrophysiological properties of the iPSC-derived BFCNs. The functional properties of the BFCN cultures were analysed by whole-cell patch clamp at week 4 of BFCN maturation. A total of 45 cells across six BFCN samples were patch-clamped, showing an average resting membrane potential of $-32 \pm 2 \mathrm{mV}$ and cell capacitance of $21 \pm 2 \mathrm{pF}$ (Figure 6A,B). Of the neurons analysed, $29 \%$ were able to fire action potentials when the membrane potential was held at $-60 \mathrm{mV}$ (Figure $6 \mathrm{C}$ ), and $36 \%$ of the cells showed aborted spikes below $0 \mathrm{mV}$. Synaptic events were also recorded in BFCN cultures (Figure 6D). 
A

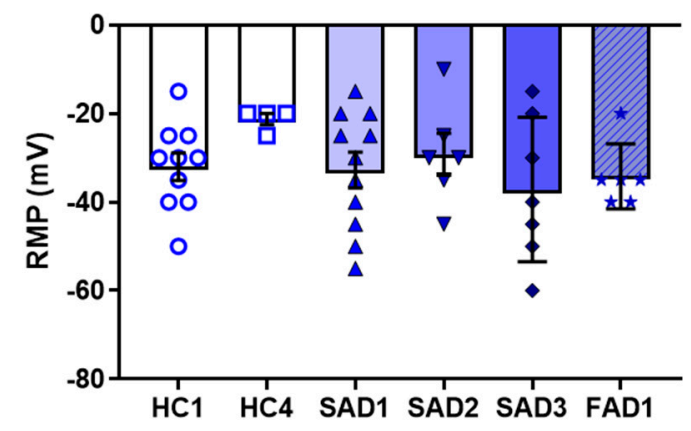

B

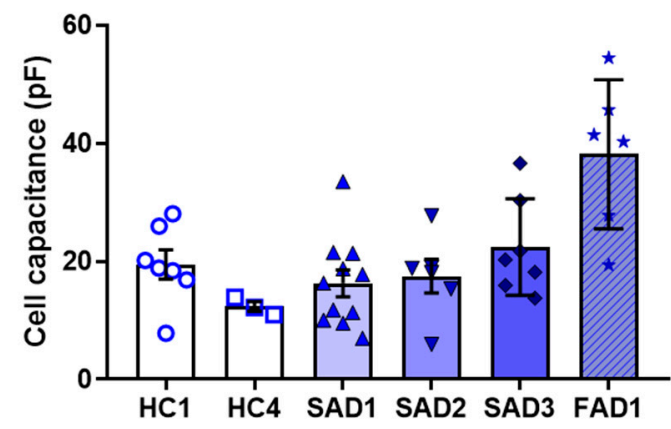

C

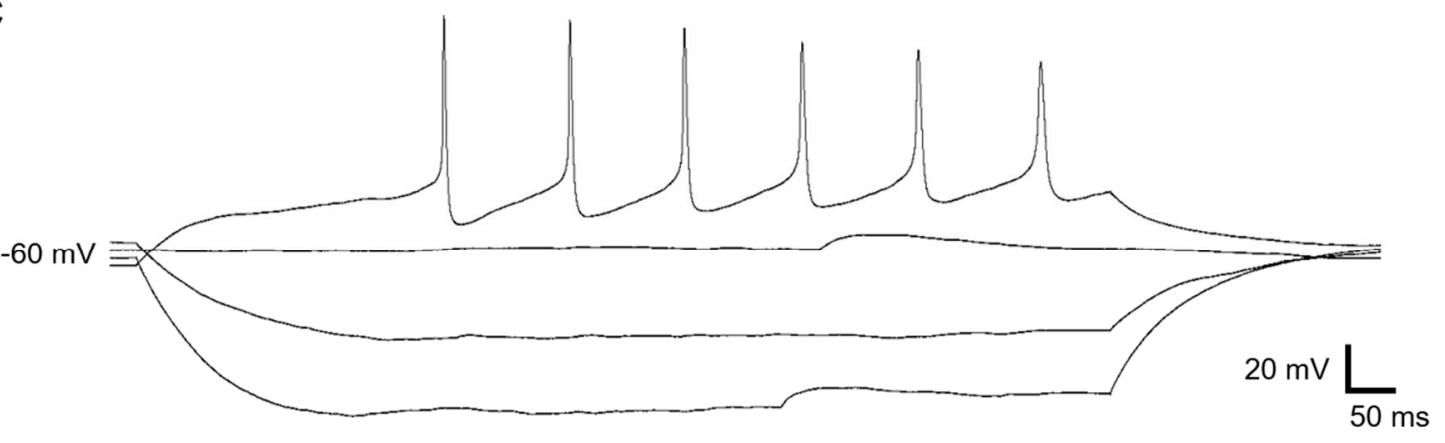

D

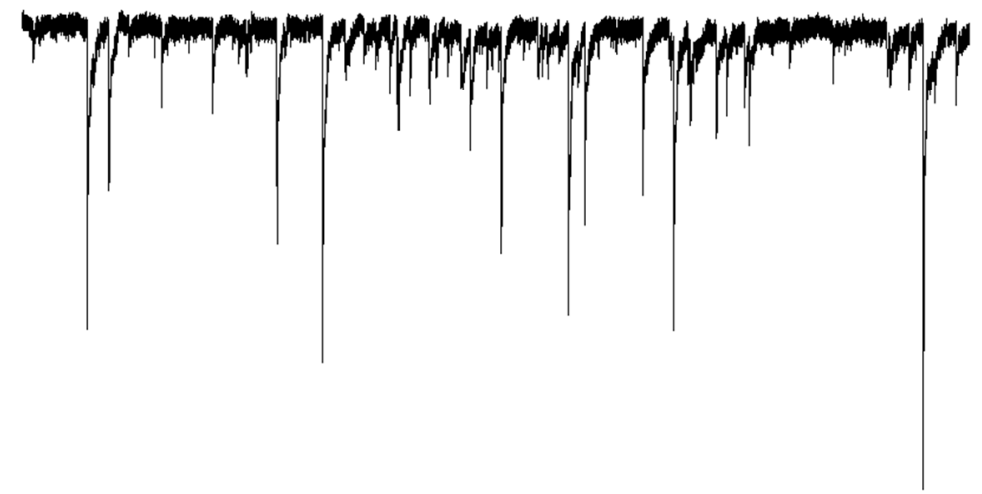

$100 \mathrm{pA} \frac{\bigsqcup_{0.1 \mathrm{~s}}}{}$

Figure 6. Electrophysiology data of BFCN cultures at week 4 of maturation. (A) Resting membrane potential and (B) cell capacitance. (C) Sample trace of a train of action potentials when membrane potential was held at $-60 \mathrm{mV}$. (D) Sample trace of synaptic events. Data in A and B were derived from $6 \mathrm{BFCN}$ differentiated samples. Individual data points representing individual cells are shown. Histogram bars represent mean values and error bars represent S.E.M.

\section{Discussion}

The generation of BFCNs from iPSCs can provide an important model to understand neurodevelopment or the neurobiology of $\mathrm{AD}$ and FTD, as this neuronal population is drastically reduced in AD and FTD patients [2]. This work establishes a reliable protocol to differentiate BFCNs cultures as shown here by differentiation of multiple cell lines by mimicking the in vivo signals required in development to generate BFCNs. The protocol relies on the use of small molecule inhibitors and growth factors avoiding transfection or cell sorting as other published protocols [11-13] to achieve a BFCN culture that expresses the characteristic markers of cholinergic neurons and negligible contamination with GFAP positive astrocytes (Figure 5). 
The use of the nCounter SPRINT System (Nanostring) allows the simultaneous analysis of a large number of genes from the same pool of cells, ensuring a reliable characterization of the BFCNs. Here were show the gene expression of markers from different stages of neuronal differentiation as a relative expression of the BFCN cultures normalised to iPSCs (Figures 3 and 4). Moreover, the relative amount of gene expression for each individual sample after housekeeper gene normalization is provided (Figures S4-S10).

Neural developmental genes showed higher expression levels in the BFCNs compared to the iPSCs (Figure 3), except EMX1, a transcription factor related with the development of the cerebral cortex and not the forebrain region [26,27], that showed no significantly different expression in BFCNs when compared to iPSCs. ASCL1, a key gene expressed in neural progenitor cells that promotes the cell cycle exit $[28,29]$, and $D L X 1$ and $D L X 2$, regulated by ASCL1 in the forebrain [30] showed increased expression in BFCN cultures when compared to iPSCs. SOX1 and PAX6, another two important transcription factors for the development of neuroectodermal commitment, and specific cholinergic progenitor markers such as FOXG1 and ISL1, required for forebrain fate [31,32] and NKX2-1, that determines the medial ganglion eminence fate, showed higher expression in BFCN cultures when compared to iPSCs. However, LHX8 did not show a significant difference in BFCN and iPSC cultures, even if three of the BFCN samples showed higher expression (Figure 3K); but it has been shown that some subsets of cholinergic neurons can be generated in the absence of Lhx8 [33]. Overall, the expression of neuronal developmental markers and specific BFCN developmental markers indicates the expected fate towards $\mathrm{BFCN}$ neurons of the cells in culture.

From all the general neuronal markers analysed (Figure 4A-J), only DAT1, a dopamine transporter, did not show a significant increase in BFCN cultures when compared to iPSCs. All neuronal markers, including TUBB3 and TH expression, the dendritic marker $M A P 2$, the pre- and post-synaptic markers SYN1 and PSD95 and the glutamate receptors GAD2, GRIA1, GRIA2 and GRIN1 showed a significant increase in BFCN cultures when compared to iPSCs. However, to ensure that specific BFCNs were generated in culture, the specific BFCN markers related to the synthesis and transport of ACh were analysed (Figure $4 \mathrm{~K}-\mathrm{P}$ ). ACHE, the choline transporter $\mathrm{CHT1}$ and the high affinity NGF receptor TRKA were upregulated; whereas CHAT, the low affinity NGF receptor NGFR, and VACHT did not show a significant difference between BFCN and iPSC cultures. However, previous studies have shown that NGFR plays a role in pluripotency [34], justifying its presence in iPSC cultures. Moreover, double immunocytochemistry of ChAT and $\beta$-III-tubulin showed an efficiency of $87 \%$ ChAT positive neurons (Figure 2A-C) indicating the cholinergic fate of the cultures.

Astrocytic markers were analysed to determine the purity of the BFCN cultures. From the four astrocytic markers analysed, ALDOC and NFIA did not show a significant difference, whereas $S 100 B$ was upregulated and EAAT1 was downregulated in the BFCN culture when compared to the iPSCs (Figure 5A-D). Given that immunocytochemistry could not identify GFAP positive staining in the cultures (Figure 5E-G), and mRNA counts of astrocyte markers were low, this indicates that the current protocol generates a highly pure culture of BFCNs.

This protocol was developed to allow for analysis of neurons, without contamination from astrocytes, as shown by the lack of GFAP staining and other astrocyte markers. Analysis of the functional properties of the BFCNs was determined by whole cell patch clamp, with neurons showing action potentials and synaptic events when the membrane potential was held at $-60 \mathrm{mV}$ (Figure $6 \mathrm{C}, \mathrm{D}$ ). Whereas recent publications have shown a resting membrane potential values of $-40 \mathrm{mV}$ in differentiated BFCNs $[13,35]$, in this study the average resting membrane potential was $-30 \mathrm{mV}$ which, taken together with the high expression of some developmental markers, show the immaturity of the neurons. An increase in the maturation time over the current 4 weeks of the protocol or the presence of other cell types, such as astrocytes, would improve maturation of neurons in culture [36]. Analysis of co-cultures can be the subject of future research.

AD has mostly been studied in the late stages to understand the pathology of the disease. However, aging in a dish remains a limitation (as reviewed in [37]), even if some studies have already shown 
different approaches that may be used to model late-onset diseases. Manipulation of telomerases [38] or treatment with progerin [39] have induced the aging phenotype in iPSC-derived neurons modelling Parkinson's disease (PD). Like PD, AD is a neurodegenerative disease whereby deposition of amyloid plaques or neurofibrillary tangles starts decades before the cognitive symptoms appear. Post mortem tissue from patients with the Lewy body variant of $\mathrm{AD}$ (dementia with Lewy bodies) also show a loss of cholinergic neurons and reduced ChAT activity very early in the disease course [40]. The iPSC-derived BFCNs can therefore help us understand the underlying pathological mechanisms that occur in the early stages of a range of neurodegenerative diseases.

\section{Conclusions}

The generation and characterization of a robust protocol to generate BFCNs from iPSCs has been described in this study. The BFCNs generated open a new way to unravel the specific neuropathology of BFCNs in AD and FTD.

Supplementary Materials: The following are available online at http://www.mdpi.com/2073-4409/9/9/2018/s1. Figure S1. Confirmation of pluripotency of iPSCs MBE2960 healthy control; Figure S2. Confirmation of pluripotency and three germ layer differentiation of iPSCs RB7-11 late-onset Alzheimer's disease (UOWi006-A); Figure S3. Confirmation of pluripotency and three germ layer differentiation of iPSCs C-10 FTD/ALS (UOWi008-A); Figure S4. RNA molecule count of housekeeper genes. iPSCs, NPCs and BFCNs samples were analysed by nCounter (Nanostring) and results are shown as RNA molecule count after internal quality control and normalisation to a reference sample used on the PlexSet; Figure S5. RNA molecule count of pluripotency markers. iPSCs, NPCs and BFCNs samples were analysed by nCounter (Nanostring) and results are shown as RNA molecule count after normalisation of total amount of RNA molecules to the housekeeper genes. The pluripotency markers NANOG and POU5F1 were analysed; Figure S6. RNA molecule count of developmental markers. iPSCs, NPCs and BFCNs samples were analysed by nCounter (Nanostring) and results are shown as RNA molecule count after normalisation of total amount of RNA molecules to the housekeeper genes. The developmental markers ASLC1, DLX1, DLX2, EMX1, PAX6 and SOX1 were analysed; Figure S7. RNA molecule count of cholinergic developmental markers. iPSCs, NPCs and BFCNs samples were analysed by nCounter (Nanostring) and results are shown as RNA molecule count after normalisation of total amount of RNA molecules to the housekeeper genes. The cholinergic developmental markers FOXG1, ISL1, LHX8 and NKX2-1 were analysed; Figure S8. RNA molecule count of neuronal markers. iPSCs, NPCs and BFCNs samples were analysed by nCounter (Nanostring) and results are shown as RNA molecule count after normalisation of total amount of RNA molecules to the housekeeper genes. The neuronal markers SLC6A3 (DAT1), GAD2, GRIA1, GRIA2, GRIN1, MAP2, DLG4 (PSD95), SYN1, TH and TUBB3 were analysed; Figure S9. RNA molecule count of cholinergic neuron markers. iPSCs, NPCs and BFCNs samples were analysed by nCounter (Nanostring) and results are shown as RNA molecule count after normalisation of total amount of RNA molecules to the housekeeper genes. The cholinergic neuronal markers ACHE, CHAT, SLC5A7 (CHT1), NGFR, NTRK1 (TRKA) and SLC18A3 (VACHT) were analysed; Figure S10. RNA molecule count of astrocytic markers. iPSCs, NPCs and BFCNs samples were analysed by nCounter (Nanostring) and results are shown as RNA molecule count after normalisation of total amount of RNA molecules to the housekeeper genes. The astrocytic markers ALDOC, SLC1A3 (EAAT1), NFIA and S100B were analysed; Table S1. List of genes analysed in Nanostring for iPSC and BFCN cultures.

Author Contributions: S.S.M.—data generation, data analysis, intellectual input, manuscript writing; M.E.-data generation, data analysis, intellectual input; R.B. - data generation, data analysis; D.D.-H.- data generation, data analysis; M.C.C.-d.-S. - data generation, intellectual input; D.H. - cell line generation; T.B.- data generation; J.A.F.—data generation; N.G.—data generation; S.Y.-provided patient samples; I.P.B.-provided patient samples; G.N.—provided patient samples; A.L.C.—provided patient samples; A.W.H.—-provided patient samples; A.P. - supervision, funding; L.O.—-funding, intellectual input, manuscript writing, supervision. All authors edited the manuscript. All authors have read and agree to the published version of the manuscript.

Funding: The research was funded by National Health and Medical Research Council (NHMRC) of Australia grants (APP1071250, and APP1095215) and a NHMRC Boosting Dementia Research Leadership Fellowship awarded to LO (APP1135720). AP is supported by a NHMRC Senior Research Fellowship (1154389).

Acknowledgments: The authors thank the donors for their participation in this research.

Conflicts of Interest: The authors declare no conflict of interest. 


\section{References}

1. Mufson, E.J.; Counts, S.E.; Perez, S.E.; Ginsberg, S.D. Cholinergic system during the progression of Alzheimer's disease: Therapeutic implications. Expert Rev. Neurother. 2008, 8, 1703-1718. [CrossRef] [PubMed]

2. Whitehouse, P.J.; Price, D.L.; Struble, R.G.; Clark, A.W.; Coyle, J.T.; Delon, M.R. Alzheimer's disease and senile dementia: Loss of neurons in the basal forebrain. Science 1982, 215, 1237-1239. [CrossRef]

3. Yiannopoulou, K.G.; Papageorgiou, S.G. Current and future treatments for Alzheimer's disease. Adv. Neurol. Disord. 2013, 6, 19-33. [CrossRef]

4. Schaeverbeke, J.; Evenepoel, C.; Bruffaerts, R.; Van Laere, K.; Bormans, G.; Dries, E.; Tousseyn, T.; Nelissen, N.; Peeters, R.; Vandenbulcke, M.; et al. Cholinergic depletion and basal forebrain volume in primary progressive aphasia. Neuroimage Clin. 2017, 13, 271-279. [CrossRef] [PubMed]

5. Convery, R.S.; Neason, M.R.; Cash, D.M.; Cardoso, M.J.; Modat, M.; Ourselin, S.; Warren, J.D.; Rohrer, J.D.; Bocchetta, M. Basal forebrain atrophy in frontotemporal dementia. Neuroimage Clin. 2020, 26, 102210. [CrossRef] [PubMed]

6. St George-Hyslop, P.H.; Petit, A. Molecular biology and genetics of Alzheimer's disease. C. R. Biol. 2005, 328, 119-130. [CrossRef] [PubMed]

7. Corder, E.H.; Saunders, A.M.; Strittmatter, W.J.; Schmechel, D.E.; Gaskell, P.C.; Small, G.W.; Roses, A.D.; Haines, J.L.; Pericak-Vance, M.A. Gene dose of apolipoprotein E type 4 allele and the risk of Alzheimer's disease in late onset families. Science 1993, 261, 921-923. [CrossRef]

8. Saunders, A.M.; Strittmatter, W.J.; Schmechel, D.; George-Hyslop, P.H.; Pericak-Vance, M.A.; Joo, S.H.; Rosi, B.L.; Gusella, J.F.; Crapper-MacLachlan, D.R.; Alberts, M.J. Association of apolipoprotein E allele epsilon 4 with late-onset familial and sporadic Alzheimer's disease. Neurology 1993, 43, 1467-1472. [CrossRef]

9. Strittmatter, W.J.; Saunders, A.M.; Schmechel, D.; Pericak-Vance, M.; Enghild, J.; Salvesen, G.S.; Roses, A.D. Apolipoprotein E: High-avidity binding to beta-amyloid and increased frequency of type 4 allele in late-onset familial Alzheimer disease. Proc. Natl. Acad. Sci. USA 1993, 90, 1977-1981. [CrossRef]

10. Engel, M.; Do-Ha, D.; Muñoz, S.S.; Ooi, L. Common pitfalls of stem cell differentiation: A guide to improving protocols for neurodegenerative disease models and research. Cell. Mol. Life Sci. 2016, 73, 3693-3709. [CrossRef]

11. Bissonnette, C.J.; Lyass, L.; Bhattacharyya, B.J.; Belmadani, A.; Miller, R.J.; Kessler, J.A. The controlled generation of functional basal forebrain cholinergic neurons from human embryonic stem cells. Stem Cells 2011, 29, 802-811. [CrossRef] [PubMed]

12. Moreno, C.L.; Della Guardia, L.; Shnyder, V.; Ortiz-Virumbrales, M.; Kruglikov, I.; Zhang, B.; Schadt, E.E.; Tanzi, R.E.; Noggle, S.; Buettner, C.; et al. iPSC-derived familial Alzheimer's PSEN2 N141I cholinergic neurons exhibit mutation-dependent molecular pathology corrected by insulin signaling. Mol. Neurodegener. 2018, 13, 33. [CrossRef] [PubMed]

13. Ortiz-Virumbrales, M.; Moreno, C.L.; Kruglikov, I.; Marazuela, P.; Sproul, A.; Jacob, S.; Zimmer, M.; Paull, D.; Zhang, B.; Schadt, E.E.; et al. CRISPR/Cas9-Correctable mutation-related molecular and physiological phenotypes in iPSC-derived Alzheimer's PSEN2 N141I neurons. Acta Neuropathol. Commun. 2017, 5, 77. [CrossRef] [PubMed]

14. Balez, R.; Steiner, N.; Engel, M.; Muñoz, S.S.; Lum, J.S.; Wu, Y.; Wang, D.; Vallotton, P.; Sachdev, P.; O'Connor, M.; et al. Neuroprotective effects of apigenin against inflammation, neuronal excitability and apoptosis in an induced pluripotent stem cell model of Alzheimer's disease. Sci. Rep. 2016, 6, 31450. [CrossRef] [PubMed]

15. Muñoz, S.S.; Balez, R.; Castro Cabral-da-Silva, M.E.; Berg, T.; Engel, M.; Bax, M.; Do-Ha, D.; Stevens, C.H.; Greenough, M.; Bush, A.; et al. Generation and characterization of human induced pluripotent stem cell lines from a familial Alzheimer's disease PSEN1 A246E patient and a non-demented family member bearing wild-type PSEN1. Stem Cell Res. 2018, 31, 227-230. [CrossRef] [PubMed]

16. Engel, M.; Balez, R.; Muñoz, S.S.; Cabral-da-Silva, M.C.; Stevens, C.H.; Bax, M.; Do-Ha, D.; Sidhu, K.; Sachdev, P.; Ooi, L. Viral-free generation and characterization of a human induced pluripotent stem cell line from dermal fibroblasts. Stem Cell Res. 2018, 32, 135-138. [CrossRef]

17. Konttinen, H.; Cabral-da-Silva, M.E.C.; Ohtonen, S.; Wojciechowski, S.; Shakirzyanova, A.; Caligola, S.; Giugno, R.; Ishchenko, Y.; Hernández, D.; Fazaludeen, M.F.; et al. PSEN1 $\triangle$ E9, APPswe, and APOE4 Confer Disparate Phenotypes in Human iPSC-Derived Microglia. Stem Cell Rep. 2019, 13, 669-683. [CrossRef] 
18. Ooi, L.; Sidhu, K.; Poljak, A.; Sutherland, G.; O'Connor, M.D.; Sachdev, P.; Münch, G. Induced pluripotent stem cells as tools for disease modelling and drug discovery in Alzheimer's disease. J. Neural Transm. 2013, 120, 103-111. [CrossRef]

19. Li, X.-J.; Zhang, X.; Johnson, M.A.; Wang, Z.-B.; Lavaute, T.; Zhang, S.-C. Coordination of sonic hedgehog and Wnt signaling determines ventral and dorsal telencephalic neuron types from human embryonic stem cells. Development 2009, 136, 4055-4063. [CrossRef]

20. Takahashi, H.; Liu, F.-C. Genetic patterning of the mammalian telencephalon by morphogenetic molecules and transcription factors. Birth Defects Res. C Embryo Today 2006, 78, 256-266. [CrossRef]

21. Alderson, R.F.; Alterman, A.L.; Barde, Y.A.; Lindsay, R.M. Brain-derived neurotrophic factor increases survival and differentiated functions of rat septal cholinergic neurons in culture. Neuron 1990, 5, $297-306$. [CrossRef]

22. Auld, D.S.; Mennicken, F.; Quirion, R. Nerve growth factor rapidly induces prolonged acetylcholine release from cultured basal forebrain neurons: Differentiation between neuromodulatory and neurotrophic influences. J. Neurosci. 2001, 21, 3375-3382. [CrossRef] [PubMed]

23. Sofroniew, M.V.; Howe, C.L.; Mobley, W.C. Nerve growth factor signaling, neuroprotection, and neural repair. Annu. Rev. Neurosci. 2001, 24, 1217-1281. [CrossRef] [PubMed]

24. van Laar, J.A.; Rustum, Y.M.; Ackland, S.P.; van Groeningen, C.J.; Peters, G.J. Comparison of 5-fluoro-2'-deoxyuridine with 5-fluorouracil and their role in the treatment of colorectal cancer. Eur. J. Cancer 1998, 34, 296-306. [CrossRef]

25. Xia, Z.; Wiebe, L.I.; Miller, G.G.; Knaus, E.E. Synthesis and biological evaluation of butanoate, retinoate, and bis(2,2,2-trichloroethyl)phosphate derivatives of 5-fluoro-2'-deoxyuridine and 2',5-difluoro-2'-deoxyuridine as potential dual action anticancer prodrugs. Arch. Pharm. 1999, 332, $286-294$. [CrossRef]

26. Gulisano, M.; Broccoli, V.; Pardini, C.; Boncinelli, E. Emx1 and Emx2 show different patterns of expression during proliferation and differentiation of the developing cerebral cortex in the mouse. Eur. J. Neurosci. 1996, 8, 1037-1050. [CrossRef]

27. Yoshida, M.; Suda, Y.; Matsuo, I.; Miyamoto, N.; Takeda, N.; Kuratani, S.; Aizawa, S. Emx1 and Emx2 functions in development of dorsal telencephalon. Development 1997, 124, 101-111.

28. Castro, D.S.; Martynoga, B.; Parras, C.; Ramesh, V.; Pacary, E.; Johnston, C.; Drechsel, D.; Lebel-Potter, M.; Garcia, L.G.; Hunt, C.; et al. A novel function of the proneural factor Ascl1 in progenitor proliferation identified by genome-wide characterization of its targets. Genes Dev. 2011, 25, 930-945. [CrossRef]

29. Ross, S.E.; Greenberg, M.E.; Stiles, C.D. Basic helix-loop-helix factors in cortical development. Neuron 2003, 39, 13-25. [CrossRef]

30. Poitras, L.; Ghanem, N.; Hatch, G.; Ekker, M. The proneural determinant MASH1 regulates forebrain Dlx1/2 expression through the I12b intergenic enhancer. Development 2007, 134, 1755-1765. [CrossRef]

31. Cho, H.-H.; Cargnin, F.; Kim, Y.; Lee, B.; Kwon, R.-J.; Nam, H.; Shen, R.; Barnes, A.P.; Lee, J.W.; Lee, S.; et al. Isl1 directly controls a cholinergic neuronal identity in the developing forebrain and spinal cord by forming cell type-specific complexes. PLoS Genet. 2014, 10, e1004280. [CrossRef] [PubMed]

32. Manuel, M.; Martynoga, B.; Yu, T.; West, J.D.; Mason, J.O.; Price, D.J. The transcription factor Foxg1 regulates the competence of telencephalic cells to adopt subpallial fates in mice. Development 2010, 137, 487-497. [CrossRef] [PubMed]

33. Zhao, Y.; Marín, O.; Hermesz, E.; Powell, A.; Flames, N.; Palkovits, M.; Rubenstein, J.L.R.; Westphal, H. The LIM-homeobox gene Lhx8 is required for the development of many cholinergic neurons in the mouse forebrain. Proc. Natl. Acad. Sci. USA 2003, 100, 9005-9010. [CrossRef]

34. Tomellini, E.; Lagadec, C.; Polakowska, R.; Le Bourhis, X. Role of p75 neurotrophin receptor in stem cell biology: More than just a marker. Cell. Mol. Life Sci. 2014, 71, 2467-2481. [CrossRef] [PubMed]

35. Yue, W.; Li, Y.; Zhang, T.; Jiang, M.; Qian, Y.; Zhang, M.; Sheng, N.; Feng, S.; Tang, K.; Yu, X.; et al. ESC-derived basal forebrain cholinergic neurons ameliorate the cognitive symptoms associated with Alzheimer's disease in mouse models. Stem Cell Rep. 2015, 5, 776-790. [CrossRef]

36. Hu, Y.; Qu, Z.; Cao, S.; Li, Q.; Ma, L.; Krencik, R.; Xu, M.; Liu, Y. Directed differentiation of basal forebrain cholinergic neurons from human pluripotent stem cells. J. Neurosci. Methods 2016, 266, 42-49. [CrossRef] 
37. Mertens, J.; Reid, D.; Lau, S.; Kim, Y.; Gage, F.H. Aging in a dish: iPSC-derived and directly induced neurons for studying brain Aging and age-related neurodegenerative diseases. Annu. Rev. Genet. 2018, 52, 271-293. [CrossRef]

38. Vera, E.; Bosco, N.; Studer, L. Generating Late-Onset Human iPSC-Based Disease Models by Inducing Neuronal Age-Related Phenotypes through Telomerase Manipulation. Cell Rep. 2016, 17, 1184-1192. [CrossRef]

39. Miller, J.D.; Ganat, Y.M.; Kishinevsky, S.; Bowman, R.L.; Liu, B.; Tu, E.Y.; Mandal, P.K.; Vera, E.; Shim, J.; Kriks, S.; et al. Human iPSC-based modeling of late-onset disease via progerin-induced aging. Cell Stem Cell 2013, 13, 691-705. [CrossRef]

40. Tiraboschi, P.; Hansen, L.A.; Alford, M.; Merdes, A.; Masliah, E.; Thal, L.J.; Corey-Bloom, J. Early and widespread cholinergic losses differentiate dementia with Lewy bodies from Alzheimer disease. Arch. Gen. Psychiatry 2002, 59, 946-951. [CrossRef]

(C) 2020 by the authors. Licensee MDPI, Basel, Switzerland. This article is an open access article distributed under the terms and conditions of the Creative Commons Attribution (CC BY) license (http://creativecommons.org/licenses/by/4.0/). 